\title{
Far-infrared spectroscopic observations of the Galactic center region $\star$
}

\author{
A. Yasuda ${ }^{1,2}$, T. Nakagawa ${ }^{1}$, M. Spaans ${ }^{3}$, Y. Okada $^{1}$, and H. Kaneda ${ }^{1}$ \\ 1 Institute of Space and Astronautical Science, Japan Aerospace Exploration Agency, 3-1-1 Yoshinodai, Sagamihara, \\ Kanagawa 229-8510, Japan \\ e-mail: yasuda@ir.isas.jaxa.jp \\ 2 The Graduate University for Advanced Studies, Shonan Village, Hayama, Kanagawa 240-193, Japan \\ 3 Kapteyn Astronomical Institute, PO Box 800, 9700 AV Groningen, The Netherlands
}

Received 28 August 2007 / Accepted 11 December 2007

\begin{abstract}
Context. The Galactic center region is luminous in the far-infrared (FIR), but dominant sources of this luminosity are still controversial.

Aims. We investigate physical conditions of the diffuse interstellar medium in the Galactic center region by multi-line spectroscopy in the FIR.

Methods. We analyze the archival data of the Galactic center region obtained with the Long-Wavelength Spectrometer on board the Infrared Space Observatory. We detect strong continuum emission and fine-structure emission lines from photo-dissociation and $\mathrm{H}$ II regions, including [O I] and [C II] lines that are dominant coolants of interstellar clouds.

Results. The observations show that the $[\mathrm{C} \mathrm{II}] /$ FIR ratio is systematically low whereas the $[\mathrm{OI}] /$ FIR ratio is almost constant toward the Galactic center region. By using a photo-dissociation region model with observed FIR parameters, we obtain radiation an effective temperature of $6600 \pm 300 \mathrm{~K}$ in the Galactic center region, which is significantly lower than $8600 \pm 500 \mathrm{~K}$ in the Galactic disk region. Conclusions. Dominant sources of the FIR luminosity in the Galactic center region are not likely to be young OB stars but rather cool stars, $\mathrm{K}$ and $\mathrm{M}$ giants, which implies that the current star-formation activity is rather low in the Galactic center region.
\end{abstract}

Key words. Galaxy: center - ISM: clouds - ISM: general - infrared: ISM

\section{Introduction}

The Galactic center region is luminous in the far-infrared (FIR). There are many pieces of observational evidence for current starformation activity in the Galactic center region. The presence of clusters of young, massive stars such as the Central, Quintuplet, and Arches clusters (e.g. Morris \& Serabyn 1996; Cotera et al. 1996; Figer et al. 1999) suggests recent star-formation activity. Well-studied star-forming clouds, such as the Sgr B2, Sgr C, Sgr D, and the $50 \mathrm{~km} \mathrm{~s}^{-1}$ clouds in the Sgr A, also show that the star-formation activity in the Galactic center region is high. Recently Goicoechea et al. (2004) observed the Sgr B2 region in the FIR fine structure emission lines, [N II], [N III], [O III], [C II], and $[\mathrm{OI}]$, and obtained the density of $n_{\mathrm{H}}=10^{3}-10^{4} \mathrm{~cm}^{-3}$ and the far-UV (FUV) radiation field of $G_{0}=10^{3}-10^{4}$, where $G_{0}$ is given in units of the value in the solar neighborhood $(1.6 \times$ $10^{-3} \mathrm{erg} \mathrm{cm}^{-2} \mathrm{~s}^{-1}$; Habing 1968). They concluded that the local radiation field in the Sgr B2 region is characterized by a hard ionizing continuum typical of an $\mathrm{O} 7$ star with a radiation effective temperature $T_{\text {eff }}$ of about $36000 \mathrm{~K}$.

On the other hand, as shown in Cox \& Laureijs (1989), the infrared excess, which is defined as infrared luminosity over Lyman alpha luminosity and is an indicator of the star-formation

* This work is based on observations with ISO, an ESA project with instruments funded by ESA Member States (especially the PI countries: France, Germany, the Netherlands and the United Kingdom) and with the participation of ISAS and NASA. activity, is $\sim 30$ in the Galactic center region, while it is $\sim 10$ in typical H II regions of the Galactic disk. This suggests that star formation in the Galactic center region is rather inactive as compared with that in the disk region, and thus dominant heating sources for the dust in the Galactic center region might not be young OB stars but rather cool stars, $\mathrm{K}$ and $\mathrm{M}$ giants (Cox \& Laureijs 1989). Odenwald \& Fazio (1984) showed that the infrared excess of a FIR extended component is 50 in the Galactic center, which implies an even later population of stars in the extended component than in FIR discrete sources that have an average value of 16. Lis et al. (2001) concluded that giant molecular clouds (GMCs) in the ridge between the Galactic center radio arc and the Sgr B clouds are externally heated by the diffuse interstellar radiation field with no evidence for the presence of luminous internal heating sources, i.e., ongoing high-mass star-formation associated with the GMCs, in spite of their large molecular masses. Furthermore, the above young cluster generation of stars seems to be specific to the very central region of the Galaxy, which does not permeate the Galactic bulge (van Loon et al. 2003). These pieces of information suggest that the general star-formation activity in the Galactic center might be low, although this region contains active star-forming regions. Hence, dominant sources of the FIR luminosity in the Galactic center region are still controversial.

FIR wavelength regions are affluent in spectral emission lines of great importance for the study of interstellar physics and chemistry, which is affected by the star-formation 


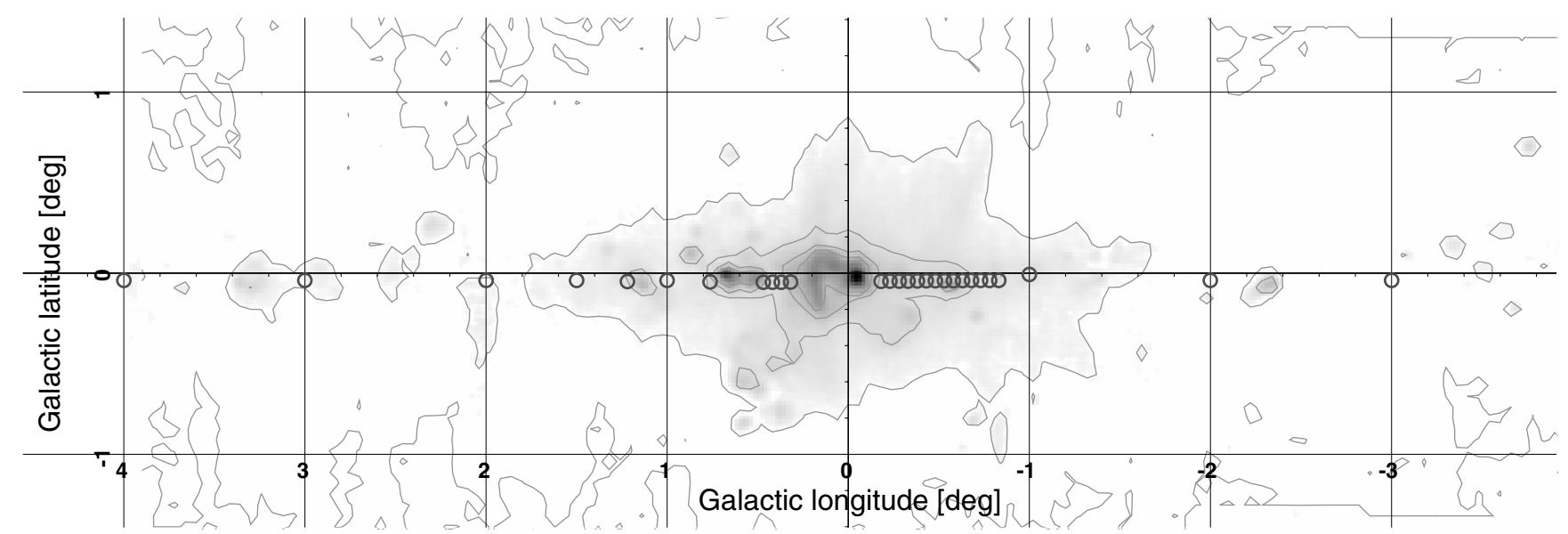

Fig. 1. ISO/LWS observational points in the Galactic center region, plotted on the $10 \mathrm{GHz}$ radio continuum map (Handa et al. 1987) with contours drawn at levels of $2.0,1.125,0.5$, and $0.125 \mathrm{~K}$. The disk regions are not shown in this figure.

activity. The COBE/FIRAS showed the large-scale distributions of such FIR emission lines along the Galactic plane (Bennett et al. 1994; Fixsen et al. 1999). Particularly, [C II] $157.7 \mu \mathrm{m}$ $\left({ }^{2} \mathrm{P}_{3 / 2} \rightarrow{ }^{2} \mathrm{P}_{1 / 2}\right)$ and $[\mathrm{O} \mathrm{I}] 63.2 \mu \mathrm{m}\left({ }^{3} \mathrm{P}_{1} \rightarrow{ }^{3} \mathrm{P}_{2}\right)$ lines are dominant coolants for general interstellar gas. Various models of photodissociation regions (PDRs) have been presented with various physical conditions (e.g. Hollenbach et al. 1991; Wolfire et al. 1990; Hollenbach \& Tielens 1997).

One notable point is that previous Galactic [C II] line observations showed that the [C II] emission was not a dominant peak in the Galactic center (e.g. Bennett et al. 1994; Nakagawa et al. 1995, 1998). On the basis of the observations using the Balloonborne Infrared Carbon Explore (BICE) showed that the ratio of the [C II] line to the FIR continuum emission is systematically low toward the Galactic center. Nakagawa et al. (1995, 1998) suggested that the low $[\mathrm{C} \mathrm{II}] /$ FIR ratio implies that the UV radiation in the Galactic center region is softer than in the Galactic disk, supporting that the luminosity of the Galactic center region may be attributed to late-type stars. However, because the observations were only made in the $[\mathrm{C}$ II] line, they could not rule out other possibilities to explain the low [C II]/FIR ratios.

In this paper, we report results of the spectroscopic observations of general Galactic center region with the LongWavelength Spectrometer (LWS) on board the Infrared Space Observatory (ISO). Owing to the wide wavelength coverage (43 to $197 \mu \mathrm{m}$ ) of the ISO/LWS, we can study multiple finestructure lines at the same time, which will be essential to pin down the physical condition of the interstellar medium (ISM). The previous ISO observations in the Galactic center are reported by many authors, which include those of $\mathrm{H}$ II regions (Rodríguez-Fernández et al. 2005), GMCs (Lis et al. 2001), nearGalactic-center discrete objects (Cotera et al. 2005; Goicoechea et al. 2004). However, most of these observations focus on local regions, whereas we cover much larger Galactic center regions. Our targets of the observations are not particular regions, such as active H II regions, but rather typical regions in the Galactic center least contaminated by strong discrete sources. Rodríguez-Fernández et al. (2004) targeted regions similar to ours. Our primary purpose is to characterize the difference of general clouds in the Galactic center region from those in the disk region, and therefore we select not only the Galactic center but also disk regions, while Rodríguez-Fernández et al. concentrated on the Galactic center region.
The availability of multi-line spectroscopy by ISO both in the Galactic center and Galactic disk regions, together with a detailed PDR model and a star-formation history model, enables us, for the first time, to obtain clear evidence showing that the current star-formation activity in the Galactic center region is generally low and the resultant soft radiation field can unambiguously explain the $[\mathrm{C} \mathrm{II}] /$ FIR ratio deficit in the Galactic center region.

\section{Observation and data reduction}

We used the ISO/LWS archived data of the Galactic center and disk regions. We first selected the data by an observational area covering $|l|<10^{\circ}$ and $|b|<1^{\circ}$. To avoid active $\mathrm{H}$ II regions, we then utilized a radio continuum survey map (Handa et al. 1987) to identify them with brightness temperatures above $2 \mathrm{~K}$ at $10 \mathrm{GHz}$. As a result, we selected 28 observations from the center and 5 observations from the disk, which are least contaminated by the active $\mathrm{H}$ II regions; the observational points in the Galactic center region are shown in Fig. 1, while the Galactic positions of the observations with the program IDs are listed in Table 1. Hereafter, we call the area in $-3^{\circ} \leq l \leq 4^{\circ}$ the Galactic center region; outside this area is the Galactic disk region. Examples of the LWS spectra obtained from the Galactic center and disk regions are shown in Fig. 2. Since all the observational points are very close to the Galactic plane $\left(b=0^{\circ}\right)$, we do not consider Galactic latitudinal dependence in the following section.

All the observations were made in the LWS01 mode, a full grating scan, to cover the wavelength range from 43 to $197 \mu \mathrm{m}$ with $\lambda / \Delta \lambda \sim 100-300$ (Gry et al. 2003). The data were calibrated with the ISO pipeline Off-Line Processing (OLP) version 10 . The data were analyzed by using the ISO Spectral Analysis Package (ISAP). The spectrum is composed of ten subspectra, each of which is an output from one of the ten detectors. After removing glitches and averaging different spectral scans, the intensity of the signal was divided by the effective solid angle of the beam to be converted to surface brightness, and then multiplied by a correction factor for extended sources. Since the detector sub-spectra showed significant mismatches between each other, which was caused by the differences in the dark current and responsivity, the sub-spectra were shifted to the reference detector, SW3, to obtain the linear spectrum (Swinyard et al. 1996). The continuum spectrum at long wavelengths is known to 

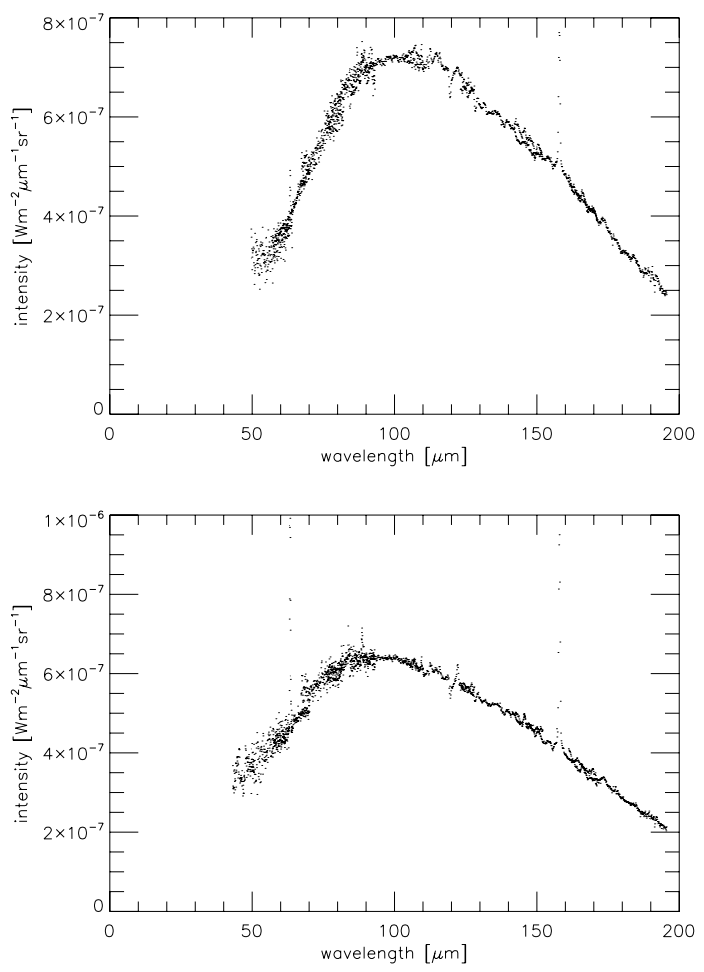

Fig. 2. Examples of the LWS spectra obtained from the Galactic center and disk regions. The spectrum of the Galactic center region (ID: 48500319) is shown in the upper panel, with that of the Galactic disk (ID: 48700703) in the lower panel.

be affected by the presence of fringes, and therefore we removed fringes using the ISAP defringe tool for extended sources. The systematic uncertainties of flux calibration are reported to be $10-20 \%$ for point sources and $50 \%$ for extended sources (Gry et al. 2003).

\section{Results}

We have detected strong continuum emission and six finestructure atomic emission lines, [O III] $52 \mu \mathrm{m}\left({ }^{3} \mathrm{P}_{2} \rightarrow{ }^{3} \mathrm{P}_{1}\right)$, [O I] $63 \mu \mathrm{m}\left({ }^{3} \mathrm{P}_{1} \rightarrow{ }^{3} \mathrm{P}_{2}\right)$, [O III] $88 \mu \mathrm{m}\left({ }^{3} \mathrm{P}_{1} \rightarrow{ }^{3} \mathrm{P}_{0}\right)$, [N II] $122 \mu \mathrm{m}\left({ }^{3} \mathrm{P}_{2} \rightarrow{ }^{3} \mathrm{P}_{1}\right)$, [O I] $145 \mu \mathrm{m}\left({ }^{3} \mathrm{P}_{0} \rightarrow{ }^{3} \mathrm{P}_{1}\right)$, and [C II] $158 \mu \mathrm{m}\left({ }^{2} \mathrm{P}_{3 / 2} \rightarrow{ }^{2} \mathrm{P}_{1 / 2}\right)$ from most of the Galactic center and disk regions. The intensities of the emission lines were obtained by Gaussian plus linear fitting. The results are summarized in Table 1.

\subsection{FIR intensity and radiation field}

We have estimated FIR continuum total intensity by fitting dust continuum spectra. We could not obtain acceptable results with single-temperature blackbody fitting, and therefore we used twotemperature blackbodies to fit the data. The total intensity radiated at a wavelength $\lambda$ is expressed as

$I_{\lambda}=\left(1-\mathrm{e}^{-\tau_{\lambda}^{\mathrm{w}}}\right) B_{\lambda}\left(T_{\mathrm{w}}\right)+\left(1-\mathrm{e}^{-\tau_{\lambda}^{\mathrm{c}}}\right) B_{\lambda}\left(T_{\mathrm{c}}\right)$,

where the upper indices $\mathrm{c}$ and $\mathrm{w}$ refer to a cold and a warm component, respectively. $B_{\lambda}(T)$ is the Planck function with temperature $T$ and dust opacity $\tau_{\lambda}$ at a wavelength $\lambda$. To obtain the dust opacity at $\lambda$, we have extrapolated the $30 \mu \mathrm{m}$ opacity, $\tau_{30}$, using a power law with an index of $\beta$ (Pierce-Price et al. 2000; Rodríguez-Fernández et al. 2004):

$\tau_{\lambda}=\tau_{30}(30 / \lambda)^{\beta}$,

where $\beta$ varies between 1 for amorphous and 2 for crystalline silicate; we used $\beta=1$ to fit the spectra.

First, we set four parameters, $T_{\mathrm{w}}, T_{\mathrm{c}}, \tau_{30}^{\mathrm{w}}$, and $\tau_{30}^{\mathrm{c}}$ to be free. However, we could not obtain stable results, and therefore we fixed $T_{\mathrm{c}}$ at $20 \mathrm{~K}$ (Mezger et al. 1989), which resulted in obtaining good fits for all the spectra (Fig. 3). We calculated the FIR intensity by integrating the best-fit blackbody curve in a wavelength range of $40 \mu \mathrm{m}$ to $120 \mu \mathrm{m}$, which is the same range used by Nakagawa et al. (1995). The fitting results and the FIR intensity thus obtained are summarized in Table 2.

We have estimated the FUV interstellar radiation field intensity $G_{0}$ by using the dust temperature, $T_{\mathrm{w}}$, derived from the above continuum fitting. We calculated $G_{0}$ by the Eq. (5) in Hollenbach et al. (1991); we assumed that the warm dust emission originates from warm surface layers with thickness $A_{\mathrm{V}} \sim 1$ of clouds illuminated by FUV radiation. The results are listed in Table 2; $G_{0}$ values in the Galactic center region exhibit a large scatter as a whole, which show somewhat larger values than those in the Galactic disk.

We estimate systematic errors of $T_{\mathrm{w}}$ and thus $G_{0}$ caused by assuming $T_{\mathrm{c}}$ is fixed at $20 \mathrm{~K}$. Rodríguez-Fernández et al. (2004) showed that clouds in the Galactic center region exhibit twotemperature dust components with variation of 14-20 K and 25-40 K from source to source. Their cold component shows a somewhat lower temperature than our assumed temperature of $20 \mathrm{~K}$ for the cold component. Hence, we have fixed $T_{\mathrm{c}}$ at $15 \mathrm{~K}$ instead of $20 \mathrm{~K}$, resulting in reducing $T_{\mathrm{w}}$ and $G_{0}$ by $2-3 \mathrm{~K}$ and a few hundred, respectively. We discuss the effect of this uncertainty below, however this uncertainty does not affect the following results significantly, because our discussion is not based on the absolute values but rather relative changes of the parameters along the Galactic plane.

\section{2. $[\mathrm{C} I I] / F I R$ and $[\mathrm{O} I] / F I R$ distributions}

From Tables 1 and 2, we calculate [C II] $157.7 \mu \mathrm{m} / \mathrm{FIR}$ and [O I] $63.2 \mu \mathrm{m} / \mathrm{FIR}$ ratios, and plot their distributions in Fig. 4.

On the basis of the BICE result, Nakagawa et al. (1995) showed that the ratio of the [C II] line to the FIR continuum emission was systematically lower in the Galactic center region than in the Galactic disk. As shown in Fig. 4, we have also clearly confirmed the systematically low [C II]/FIR ratio toward the Galactic center region, as reported in Nakagawa et al. (1995) from independent observations with different calibration. We selected the data least contaminated by active $\mathrm{H}$ II regions with the ISO/LWS beam size $\left(\sim 80^{\prime \prime}\right)$ much smaller than that $\left(\sim 15^{\prime}\right)$ of the BICE, while the BICE result was obtained by averaging data along the Galactic latitude over the range of $|b|<2^{\circ}$. In spite of such a large difference in the spatial scale of each data point, both results show an excellent agreement with each other. Small differences between the ISO and BICE results may be due to a difference in the method of estimating the FIR intensity; Nakagawa et al. (1995) used the total flux between 40 and $120 \mu \mathrm{m}$ derived from the IRAS 60 and $100 \mu \mathrm{m}$ data (Helou et al. 1988).

For comparative purposes, we also show results obtained from active $\mathrm{H}$ II regions in the same way in Fig. 4; the [C II]/FIR ratios in the active $\mathrm{H}$ II regions reveal significantly smaller values than those from our selected regions. Thus, the resultant smooth $[\mathrm{CII}] / \mathrm{FIR}$ distribution with small scatter in 
Table 1. Atomic line intensities observed in the Galactic center and disk regions.

\begin{tabular}{|c|c|c|c|c|c|c|c|c|}
\hline \multirow[t]{2}{*}{ ID } & \multicolumn{2}{|c|}{ Position (degree) } & \multicolumn{6}{|c|}{ Line Intensity $\left(10^{-8} \mathrm{~W} \mathrm{~m}^{-2} \mathrm{sr}^{-1}\right)$} \\
\hline & $\ell$ & $b$ & [O III] $51.8 \mu \mathrm{m}$ & [O I] $63.2 \mu \mathrm{m}$ & [O III] $88.4 \mu \mathrm{m}$ & [N II] $121.9 \mu \mathrm{m}$ & [O I] $145.5 \mu \mathrm{m}$ & {$[\mathrm{C} \mathrm{III} 157.7 \mu \mathrm{m}$} \\
\hline 47900823 & -6.00 & -0.04 & $1.85 \pm 0.53$ & $3.55 \pm 0.19$ & $1.40 \pm 0.22$ & $0.73 \pm 0.17$ & $0.42 \pm 0.10$ & $20.4 \pm 0.26$ \\
\hline 48500121 & -4.00 & -0.04 & $3.80 \pm 0.86$ & $3.88 \pm 0.14$ & $7.18 \pm 0.37$ & $1.92 \pm 0.19$ & $0.74 \pm 0.16$ & $27.8 \pm 0.41$ \\
\hline 48500319 & -3.00 & -0.04 & $1.06 \pm 0.20$ & $2.52 \pm 0.12$ & $1.07 \pm 0.20$ & $1.00 \pm 0.11$ & $0.90 \pm 0.06$ & $18.1 \pm 0.18$ \\
\hline 48500517 & -2.00 & -0.04 & $1.97 \pm 0.50$ & $3.45 \pm 0.091$ & $0.77 \pm 0.15$ & $2.00 \pm 0.19$ & $0.68 \pm 0.12$ & $19.7 \pm 0.18$ \\
\hline 48500727 & -1.20 & -0.30 & $0.94 \pm 0.82$ & $7.61 \pm 0.15$ & $1.22 \pm 0.48$ & $4.60 \pm 0.60$ & $1.52 \pm 0.43$ & $35.1 \pm 0.41$ \\
\hline 48500815 & -1.00 & 0.01 & $4.99 \pm 0.87$ & $1.56 \pm 0.19$ & $6.25 \pm 0.35$ & $11.2 \pm 1.2$ & $1.90 \pm 0.46$ & $56.5 \pm 0.88$ \\
\hline 32600211 & -0.83 & -0.04 & $121 \pm 5.0$ & $24.8 \pm 3.4$ & $124 \pm 5.5$ & $14.2 \pm 2.0$ & $2.77 \pm 1.4$ & $66.7 \pm 1.2$ \\
\hline 32600211 & -0.78 & -0.04 & - & $28.5 \pm 3.1$ & - & $11.4 \pm 1.1$ & $1.73 \pm 1.2$ & $75.4 \pm 4.2$ \\
\hline 32600211 & -0.73 & -0.04 & - & $26.9 \pm 3.8$ & - & $13.6 \pm 9.3$ & $2.34 \pm 1.6$ & $78.2 \pm 3.5$ \\
\hline 32600211 & -0.68 & -0.04 & - & $28.3 \pm 7.1$ & - & $16.6 \pm 1.0$ & $2.99 \pm 1.7$ & $78.5 \pm 1.8$ \\
\hline 32600211 & -0.63 & -0.04 & - & $29.1 \pm 9.1$ & - & $14.8 \pm 1.5$ & $3.55 \pm 1.2$ & $89.2 \pm 1.8$ \\
\hline 32600211 & -0.58 & -0.04 & $15.0 \pm 6.7$ & $45.2 \pm 8.8$ & - & $24.0 \pm 2.4$ & $5.82 \pm 1.2$ & $116 \pm 3.1$ \\
\hline 32600211 & -0.53 & -0.04 & - & $28.5 \pm 4.4$ & $9.08 \pm 2.4$ & $18.3 \pm 4.2$ & $4.48 \pm 1.1$ & $104 \pm 1.9$ \\
\hline 32600211 & -0.48 & -0.04 & $21.8 \pm 6.0$ & $40.0 \pm 10$ & - & $28.7 \pm 1.4$ & $4.61 \pm 1.2$ & $122 \pm 2.5$ \\
\hline 32600211 & -0.43 & -0.04 & $24.3 \pm 6.0$ & $38.2 \pm 6.0$ & - & $25.8 \pm 2.5$ & - & $113 \pm 3.0$ \\
\hline 32600211 & -0.38 & -0.04 & $20.2 \pm 8.5$ & $43.0 \pm 6.3$ & $13.2 \pm 3.5$ & $21.4 \pm 9.4$ & $6.99 \pm 1.8$ & $111 \pm 3.2$ \\
\hline 32600211 & -0.33 & -0.04 & $26.2 \pm 6.5$ & $48.7 \pm 6.2$ & $20.8 \pm 5.1$ & $21.8 \pm 8.8$ & $6.38 \pm 1.1$ & $121 \pm 2.7$ \\
\hline 32600211 & -0.28 & -0.04 & $44.7 \pm 4.9$ & $46.3 \pm 6.2$ & $36.4 \pm 8.1$ & $33.9 \pm 8.1$ & $6.19 \pm 1.8$ & $122 \pm 2.7$ \\
\hline 32600211 & -0.23 & -0.04 & $27.6 \pm 4.7$ & $38.4 \pm 7.5$ & $13.7 \pm 2.8$ & $36.3 \pm 1.3$ & $5.55 \pm 1.5$ & $111 \pm 2.6$ \\
\hline 32600211 & -0.18 & -0.04 & - & $43.6 \pm 4.8$ & $16.2 \pm 3.3$ & $23.8 \pm 8.4$ & $8.14 \pm 1.6$ & $106 \pm 2.3$ \\
\hline 32600211 & -0.13 & -0.05 & - & $62.6 \pm 5.0$ & $26.5 \pm 4.0$ & $31.1 \pm 4.6$ & $9.64 \pm 1.4$ & $122 \pm 3.4$ \\
\hline 32600211 & 0.32 & -0.05 & $11.0 \pm 3.7$ & $41.0 \pm 2.4$ & $26.0 \pm 5.8$ & $41.9 \pm 3.8$ & $9.11 \pm 0.98$ & $110 \pm 2.4$ \\
\hline 32600211 & 0.37 & -0.05 & $9.06 \pm 3.1$ & $27.9 \pm 1.6$ & $6.77 \pm 3.4$ & $13.9 \pm 2.7$ & $6.24 \pm 0.95$ & $76.8 \pm 2.5$ \\
\hline 32600211 & 0.41 & -0.05 & $14.4 \pm 4.9$ & $35.4 \pm 1.2$ & $8.66 \pm 2.7$ & $14.3 \pm 2.4$ & $6.18 \pm 1.3$ & $83.1 \pm 2.1$ \\
\hline 32600211 & 0.47 & -0.05 & $33.7 \pm 3.4$ & $88.7 \pm 3.1$ & $36.0 \pm 3.6$ & $45.6 \pm 3.5$ & $12.8 \pm 2.1$ & $151 \pm 4.7$ \\
\hline 31300507 & 0.76 & -0.05 & - & $15.1 \pm 1.3$ & $9.85 \pm 2.7$ & $8.06 \pm 2.1$ & $3.81 \pm 1.29$ & $32.1 \pm 1.7$ \\
\hline 49401011 & 1.00 & -0.04 & $2.58 \pm 0.41$ & $8.00 \pm 0.16$ & $1.48 \pm 0.13$ & $5.10 \pm 1.1$ & $1.72 \pm 0.35$ & $27.6 \pm 0.99$ \\
\hline 32600310 & 1.22 & -0.05 & $22.1 \pm 4.6$ & $11.5 \pm 4.3$ & $8.99 \pm 3.49$ & $8.13 \pm 1.4$ & - & $42.9 \pm 2.4$ \\
\hline 47001910 & 1.50 & -0.04 & $4.52 \pm 0.14$ & $8.82 \pm 0.22$ & $1.76 \pm 0.13$ & $4.56 \pm 0.79$ & $1.31 \pm 0.44$ & $26.7 \pm 0.96$ \\
\hline 47001709 & 2.00 & -0.04 & $1.00 \pm 0.17$ & $2.84 \pm 0.27$ & $0.56 \pm 0.061$ & $1.34 \pm 0.35$ & $1.31 \pm 0.30$ & $16.5 \pm 0.19$ \\
\hline 47001407 & 3.00 & -0.04 & $1.92 \pm 0.90$ & $3.41 \pm 0.26$ & $2.60 \pm 0.32$ & $5.27 \pm 0.69$ & $0.35 \pm 0.21$ & $24.0 \pm 0.29$ \\
\hline 67700205 & 4.00 & -0.04 & $2.19 \pm 0.78$ & $3.73 \pm 0.16$ & $1.29 \pm 0.41$ & $2.24 \pm 0.17$ & $0.96 \pm 0.13$ & $25.8 \pm 0.55$ \\
\hline 48700703 & 6.00 & -0.04 & $1.53 \pm 0.57$ & $18.6 \pm 0.23$ & $2.46 \pm 0.36$ & $2.55 \pm 0.15$ & $1.54 \pm 0.21$ & $34.5 \pm 0.45$ \\
\hline 49301602 & 8.00 & -0.04 & $4.64 \pm 0.43$ & $8.72 \pm 0.16$ & $9.04 \pm 0.25$ & $4.21 \pm 0.39$ & $1.06 \pm 0.17$ & $45.1 \pm 0.40$ \\
\hline 49300201 & 12.00 & -0.04 & $1.21 \pm 1.60$ & $5.28 \pm 0.17$ & $0.79 \pm 0.25$ & $2.20 \pm 0.19$ & $1.03 \pm 0.10$ & $30.6 \pm 0.50$ \\
\hline
\end{tabular}

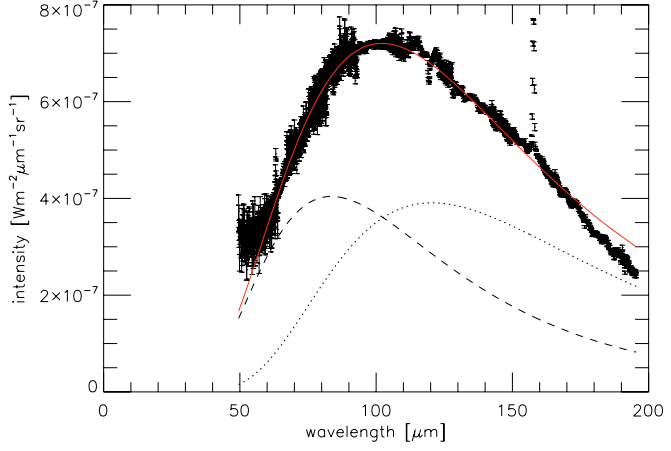

Fig. 3. Example of double-temperature blackbody model fitting to the dust continuum observed in a typical Galactic center region located at $(\ell, b) \sim(-3,0)$. The best-fit curve is shown in the solid line, while the warm and the cold component are shown in the dashed and the dotted line, respectively.

Fig. 4 will reflect successful rejection of the contribution of active $\mathrm{H}$ II regions, which in turn implies that active H II regions are not dominant FIR sources in the Galactic center region for the large beam size of the BICE; such relatively small contribution of active $\mathrm{H}$ II regions in the Galactic center region is also suggested by the infrared excess (Cox \& Laureijs 1989), as well as by the [N II] $205 \mu \mathrm{m}$ line distribution along the Galactic plane
(Bennett et al. 1994; Fixsen et al. 1999). Hence, it is very likely that our selected data safely represent typical diffuse ISM in the Galactic center region.

In contrast to the $[\mathrm{C} \mathrm{II}] / \mathrm{FIR}$ distribution, the [O I]/FIR distribution in Fig. 4 shows almost constant or even a little higher values in the center along the Galactic plane, potentially providing us with a crucial clue to interpret the suppressed [C II]/FIR ratio. The [O I] line has a significantly higher critical density than the [C II] line (Crawford et al. 1985; Genzel 1991), tracing relative high gas density regions in the Galactic center region. In addition, the [O I] line has the dependency on radiation effective temperature different from that of the $[\mathrm{C} \mathrm{II}]$ line (see below). The marked difference is utilized to discuss the condition of PDRs in the Galactic center region in the following section.

\section{Discussion}

\subsection{PDRs in the Galactic center region}

\subsubsection{What makes the $[\mathrm{C} I] / F I R$ ratio deficit?}

We discuss three possibilities to explain the systematically low [C II]/FIR ratios observed toward the Galactic center region: the dominance of a strong FUV radiation, a spectrally soft radiation field, and high gas densities. 
Table 2. Spectral fitting results together with the FIR intensities integrated over a 40-120 $\mu \mathrm{m}$ range and the interstellar radiation field $G_{0}$ derived from $T_{\mathrm{w}}$. The errors are estimated from covariance matrices.

\begin{tabular}{cccccc}
\hline \hline $\mathrm{ID}$ & $T_{\mathrm{w}}(\mathrm{K})$ & $\tau_{30}^{\mathrm{w}}\left(10^{-2}\right)$ & $\tau_{30}^{\mathrm{c}}\left(10^{-1}\right)$ & $\mathrm{FIR}\left(10^{-4} \mathrm{~W} \mathrm{~m}^{-2} \mathrm{sr}^{-1}\right)$ & $G_{0}$ \\
\hline 47900823 & $33.39 \pm 0.03$ & $0.402 \pm 0.002$ & $1.389 \pm 0.001$ & 0.34 & 1000 \\
48500121 & $33.73 \pm 0.01$ & $0.683 \pm 0.002$ & $1.466 \pm 0.001$ & 0.47 & 1000 \\
48500319 & $28.15 \pm 0.02$ & $1.95 \pm 0.01$ & $1.389 \pm 0.002$ & 0.45 & 400 \\
48500517 & $29.42 \pm 0.02$ & $1.80 \pm 0.01$ & $1.168 \pm 0.002$ & 0.48 & 500 \\
48500727 & $33.12 \pm 0.02$ & $1.56 \pm 0.01$ & $2.612 \pm 0.002$ & 0.92 & 900 \\
48500815 & $33.90 \pm 0.01$ & $3.01 \pm 0.01$ & $3.701 \pm 0.003$ & 1.76 & 1100 \\
32600211 & $38.97 \pm 0.04$ & $2.31 \pm 0.01$ & $3.52 \pm 0.01$ & 2.6 & 2100 \\
32600211 & $36.15 \pm 0.03$ & $3.81 \pm 0.02$ & $3.61 \pm 0.02$ & 2.8 & 1500 \\
32600211 & $36.05 \pm 0.02$ & $4.50 \pm 0.02$ & $3.90 \pm 0.01$ & 3.3 & 1400 \\
32600211 & $35.08 \pm 0.04$ & $5.75 \pm 0.03$ & $3.66 \pm 0.02$ & 3.5 & 1300 \\
32600211 & $34.99 \pm 0.03$ & $7.73 \pm 0.04$ & $4.17 \pm 0.02$ & 4.5 & 1200 \\
32600211 & $36.63 \pm 0.03$ & $6.47 \pm 0.03$ & $4.68 \pm 0.02$ & 4.9 & 1600 \\
32600211 & $33.89 \pm 0.03$ & $8.06 \pm 0.04$ & $5.52 \pm 0.03$ & 4.1 & 1100 \\
32600211 & $37.28 \pm 0.03$ & $6.62 \pm 0.04$ & $5.21 \pm 0.03$ & 5.5 & 1700 \\
32600211 & $38.99 \pm 0.04$ & $7.04 \pm 0.04$ & $3.92 \pm 0.03$ & 7.1 & 2100 \\
32600211 & $37.35 \pm 0.03$ & $6.99 \pm 0.04$ & $3.64 \pm 0.03$ & 5.7 & 1700 \\
32600211 & $37.82 \pm 0.03$ & $7.48 \pm 0.04$ & $3.02 \pm 0.03$ & 6.4 & 1800 \\
32600211 & $40.10 \pm 0.04$ & $5.52 \pm 0.03$ & $3.51 \pm 0.02$ & 6.5 & 2400 \\
32600211 & $37.06 \pm 0.03$ & $7.19 \pm 0.03$ & $3.53 \pm 0.02$ & 5.6 & 1700 \\
32600211 & $38.33 \pm 0.02$ & $6.59 \pm 0.02$ & $5.08 \pm 0.01$ & 6.2 & 2000 \\
32600211 & $40.82 \pm 0.04$ & $5.56 \pm 0.03$ & $5.76 \pm 0.03$ & 7.4 & 2700 \\
32600211 & $44.23 \pm 0.02$ & $4.15 \pm 0.01$ & $5.46 \pm 0.01$ & 8.2 & 4000 \\
32600211 & $35.51 \pm 0.03$ & $7.18 \pm 0.03$ & $3.91 \pm 0.02$ & 4.5 & 1300 \\
32600211 & $34.10 \pm 0.03$ & $9.05 \pm 0.05$ & $4.36 \pm 0.03$ & 4.6 & 1100 \\
32600211 & $41.43 \pm 0.04$ & $6.24 \pm 0.03$ & $6.56 \pm 0.03$ & 8.9 & 2900 \\
31300507 & $31.00 \pm 0.02$ & $3.45 \pm 0.02$ & $1.71 \pm 0.01$ & 2.5 & 700 \\
49401011 & $30.17 \pm 0.02$ & $2.03 \pm 0.01$ & $6.430 \pm 0.003$ & 1.2 & 600 \\
32600310 & $33.34 \pm 0.09$ & $1.65 \pm 0.02$ & $5.84 \pm 0.02$ & 1.3 & 1000 \\
47001910 & $32.44 \pm 0.02$ & $0.801 \pm 0.003$ & $4.459 \pm 0.001$ & 0.80 & 4000 \\
47001709 & $28.21 \pm 0.02$ & $2.14 \pm 0.01$ & $2.424 \pm 0.003$ & 0.60 & 700 \\
47001407 & $45.73 \pm 0.10$ & $0.11 \pm 0.001$ & $2.750 \pm 0.003$ & 0.57 & 300 \\
67700205 & $28.29 \pm 0.01$ & $3.03 \pm 0.01$ & $1.175 \pm 0.002$ & 0.59 & 0.55 \\
48700703 & $33.82 \pm 0.02$ & $0.561 \pm 0.003$ & $1.644 \pm 0.001$ & 0.46 & \\
49301602 & $30.98 \pm 0.02$ & $1.49 \pm 0.01$ & $1.311 \pm 0.002$ & & 400 \\
49300201 & $25.84 \pm 0.01$ & $5.23 \pm 0.02$ & $0.271 \pm 0.004$ & & \\
\hline & & & & & \\
\hline
\end{tabular}

First, we discuss the effect of a strong radiation field. The FIR emission intensity increases with $G_{0}$, while the [C II] line emission is relatively easy to saturate at large $G_{0}$ because of its low excitation energy $(E / k=92 \mathrm{~K})$. Therefore, if the low [C II]/FIR ratio is due to large $G_{0}$, the ratio should show negative correlation with $G_{0}$; Fig. 2 in Nakagawa et al. (1995) demonstrates this negative correlation in the Galactic disk region. Hence, in order to seek the possibility that strong interstellar radiation fields are responsible for the low [C II]/FIR ratios, we plot the $[\mathrm{C}$ II $] /$ FIR ratio against $G_{0}$ in Fig. 5. The result shows that the $[\mathrm{C} \mathrm{II}] /$ FIR ratio in the Galactic center region is rather almost constant over a wide range in $G_{0}$, and the ratio is significantly lower than that in the disk at similar $G_{0}$ values (Fig. 5). Therefore, it is unlikely that the $[\mathrm{C} \mathrm{II}] /$ FIR ratio can be attributed to the strong FUV radiation fields in the Galactic center region. Figure 5 shows many data points in the Galactic center region that have significantly lower $[\mathrm{C} \mathrm{II}] /$ FIR ratios than those in the disk, whereas they have similar $G_{0}$ values of $\sim 10^{3}$. In other words, the suppression of [C II]/FIR in the regions with large $G_{0}$ can be explained by the saturation of [C II] line emission (Tielens 2005). Therefore, in order to eliminate the $G_{0}$ dependence in the following discussion, we only consider the regions with $G_{0}$ smaller than 1500 .

Second, we discuss the possibility of the high density case. In high-density PDRs, the [C II] line intensity is saturated due to its low critical density $\left(n_{\mathrm{cr}} \sim 3 \times 10^{3} \mathrm{~cm}^{-3}\right.$; Crawford et al. 1985). Therefore, the dominance of high-density PDRs over low-density ones can again explain the low [C II]/FIR ratio in the Galactic center region. Hence, we estimate atomic hydrogen densities by using the ratio of the two [O I] emission lines at 63 and $145 \mu \mathrm{m}$ in the Galactic center region. The [O I] $63 \mu \mathrm{m}$ emission line is presumably not optically thin in the Galactic center region; under the assumption of the atomic gas temperature of $T=350 \mathrm{~K}$ (Genzel et al. 1985), the total hydrogen column density of $N_{\mathrm{H} \text {,tot }}=5 \times 10^{22} \mathrm{~cm}^{-2}$ (Oort 1977; Mathis 1990), the velocity dispersion of $\Delta V \sim 30 \mathrm{~km} \mathrm{~s}^{-1}$ (Genzel 1991), and the $\mathrm{O}$ abundance relative to $\mathrm{H}$ of $\delta_{\mathrm{O}}=5 \times 10^{-4}$ (Tielens $\&$ Hollenbach 1985), the optical depths of the [O I] $63 \mu \mathrm{m}$ and $145 \mu \mathrm{m}$ lines are calculated as $\tau_{63} \sim 1.6$ and $\tau_{145} \sim 0.54$, respectively. By taking this into account with an assumption that both lines are emitted from the same neutral regions lying along the line of sight, hydrogen densities $n_{\mathrm{H}}$ have been constrained to be lower than $1 \times 10^{4} \mathrm{~cm}^{-3}$ for the observational points, since the deviation from local thermal equilibrium (LTE) for level populations typically goes as $1 /\left(1+n_{\mathrm{H}} / n_{\mathrm{cr}}\right)$. This density $\left(1 \times 10^{4} \mathrm{~cm}^{-3}\right)$ does not exceed the critical density of [O I] very much. In addition, the [O I] emission lines tend to be emitted from the regions of higher densities. Hence, most Galactic center PDRs do not seem to have gas densities high enough to significantly suppress the $[\mathrm{CII}] /$ FIR ratio. And yet, the estimate of $n_{\mathrm{H}}$ from the 

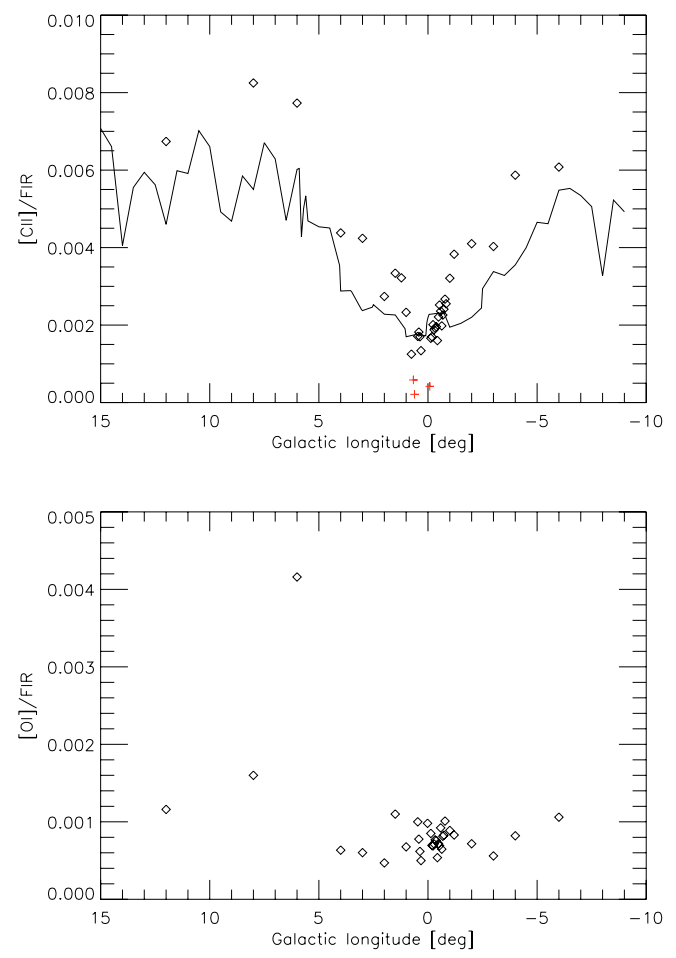

Fig. 4. Distributions of the [C II $157.7 \mu \mathrm{m} / \mathrm{FIR}$ ratio (upper panel) and the [OI] $63.2 \mu \mathrm{m} / \mathrm{FIR}$ ratio (lower panel) from the ISO results. The BICE results (Nakagawa et al. 1995) on the [C II]/FIR ratio are also shown by a solid curve in the upper panel, while the ISO results are indicated by open diamonds. The ISO results for strong H II regions are shown together by pluses for a comparative purpose. The statistical error of each data point is comparable to or smaller than the size of the symbol.

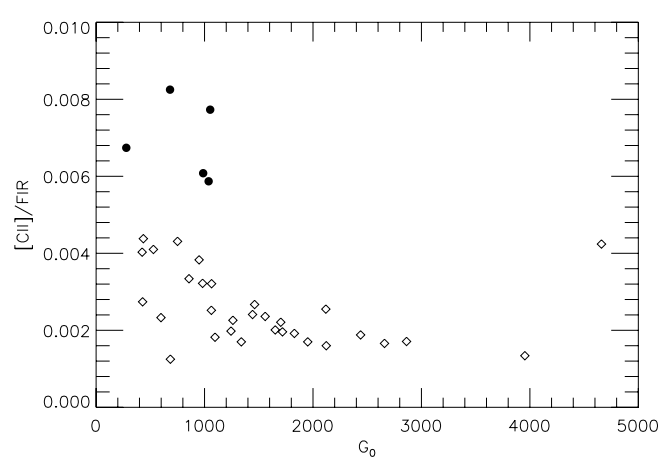

Fig. 5. Relation between the $\left[\mathrm{C}\right.$ II]/FIR ratio and $G_{0}$. The diamonds and filled circles correspond to the data in the Galactic center region and the Galactic disk, respectively.

[O I] lines alone is not conclusive to rule out the possibility of high-density PDRs for the low [C II]/FIR ratio in the Galactic center region.

The third possibility to explain the low [C II]/FIR ratios is that the interstellar radiation field may be significantly soft in the Galactic center region, as pointed out in Nakagawa et al. (1995). We have evaluated softness of the radiation fields by adopting the PDR model by Spaans et al. (1994), where they take account of color temperatures of illuminating radiation fields $\left(T_{\text {eff }}\right)$ in their calculation. On the basis of the model, we have performed detailed calculations for the chemistry and thermal balance in PDRs for various values of $n_{\mathrm{H}}$ and $G_{0}$ by changing $T_{\mathrm{eff}}$. In soft radiation fields, $\mathrm{C}^{+} / \mathrm{C} / \mathrm{CO}$ transition layers shift toward
Table 3. Derived physical conditions of the ISM.

\begin{tabular}{lccc}
\hline \hline & $G_{0}$ & $\log n_{\mathrm{H}}\left[\mathrm{cm}^{3}\right]$ & $T_{\text {eff }}[\mathrm{K}]$ \\
\hline Galactic center region & $900 \pm 400$ & $2.9 \pm 0.1$ & $6600 \pm 300$ \\
Galactic disk region & $800 \pm 300$ & $2.8 \pm 0.1$ & $8600 \pm 500$ \\
\hline
\end{tabular}

the surface of a PDR, because fewer photons are available to ionize $\mathrm{C}$ and to photodissociate $\mathrm{CO}$, making a $\mathrm{C}^{+}$layer thinner, while soft radiation field with effective temperatures of $\sim 6000 \mathrm{~K}$ (cf. $T_{\text {eff }} \sim 30000 \mathrm{~K}$ for general radiation field in the solar neighborhood; Draine 1978) still heat dust grains efficiently. On the other hand, $\mathrm{O}$ distribution in a cloud does not change very much with effective temperature, since, due to the larger abundance of $\mathrm{O}$ over $\mathrm{C}$, there is always a significant abundance of atomic oxygen in the first few magnitudes of extinction. In consequence, [C II]/FIR ratios strongly depend on effective temperatures of radiation fields, while $[\mathrm{O}$ I]/FIR ratios are less sensitive to effective temperatures.

We have already obtained the incident FUV intensity $G_{0}$, and thus we can derive $n_{\mathrm{H}}$ and effective temperature $T_{\text {eff }}$ from the PDR model by using the observed intensities of the [C II] and [O I] $63 \mu \mathrm{m}$ emission lines that have different dependences on $T_{\text {eff }}$ and $n_{\mathrm{H}}$. The FIR intensity in the model is calculated by integrating the dust emission from the warm surface layer in a wavelength range of 40 to $120 \mu \mathrm{m}$; the same range is used as when obtaining the observed FIR intensity and the same cloud configuration is assumed as that consistently adopted in the PDR model of Spaans et al. (1994). We summarize the results in Table 3.

The results show that the Galactic center regions with $G_{0}$ smaller than 1500 have radiation effective temperatures of $6600 \pm 300 \mathrm{~K}$, which are significantly lower than $8600 \pm 500 \mathrm{~K}$ in the Galactic disk regions with similar $G_{0}$ values. Hence, we have found that the interstellar radiation fields are softer in the Galactic center region, which supports the suggestion by Nakagawa et al. (1995) that the low [C II]/FIR ratio in the Galactic center region may be due to relative softness of radiation fields. The results also show that the PDRs responsible for the observed [C II]/FIR and [OI]/FIR have as small $n_{\mathrm{H}}$ values as $\sim 900 \mathrm{~cm}^{-3}$, which are well below the critical density of the [C II] line emission $\left(3 \times 10^{3} \mathrm{~cm}^{-3}\right.$; Crawford et al. 1985). The $n_{\mathrm{H}}$ values thus obtained by the PDR model are also consistent with the above result from the [O I] $63 \mu \mathrm{m} / 145 \mu \mathrm{m}$ emission line ratio. We estimate the systematic uncertainty of $T_{\text {eff }}$ caused by $G_{0}$. When $G_{0}$ changes by a few hundreds lower (or higher), the derived $T_{\text {eff }}$ changes by a few hundred Kelvin lower (or higher). Thus, the absolute values of $T_{\text {eff }}$ have uncertainties of a few hundred Kelvin $(\sim 200 \mathrm{~K})$. The relatively weak impact of the error in $G_{0}$ on our derived $T_{\text {eff }}$ is caused by the fact that the ratio between hard UV (which ionizes carbon above $11.2 \mathrm{eV}$ and heats) and soft UV (which only heats) completely drives the abundance and emissivity of $\mathrm{C}^{+}$, rather than the frequency integral over the radiation field $\left(G_{0}\right)$. Hence, significant changes in $G_{0}$ can be compensated by modest variations in $T_{\text {eff }}$. Our discussion is not based on the absolute values but on the relative changes of the parameter along the Galactic plane. On the other hand, derived $n_{\mathrm{H}}$ hardly changes with $G_{0}$. Hence, we can conclude that not higher densities but softer radiation environment in the Galactic center region is the dominant source to suppress the [C II] line intensity in the Galactic center region. 


\subsubsection{Other lines}

Our PDR model with soft radiation field also gave some predictions for other important emission lines (Spaans et al. 1994). The model predicts that species such as $\mathrm{Fe}$ and $\mathrm{Si}$ with ionization potential close to $\mathrm{C}$ show trends similar to [C II]. However, neither [Fe II] nor [Si II] have been observed in such a large scale of the Galactic center region. For molecular lines, the model also predicts that water lines are a factor of a few brighter for a soft radiation field (Spaans et al. 2004). On the other hand, the model predicts that the $[\mathrm{CI}]$ lines hardly depend on $T_{\text {eff }}$ with an increase only by a factor of $\sim 1.5$ for the cool PDRs. The COBE observations showed both the [C I] $609 \mu \mathrm{m}$ and $370 \mu \mathrm{m}$ are of comparable brightness in the Galactic center and the disk (Fixsen et al. 1999; Bennett et al. 1994), which is again compatible with our result.

\subsubsection{Warm molecular clouds}

Galactic center clouds are known to differ from Galactic disk clouds in their physical and chemical characteristics. One of the still open crucial issues is the difference in temperature of molecular clouds; gas temperatures of typical molecular clouds in the Galactic center region are systematically higher $(T \sim 70 \mathrm{~K}$; Morris \& Serabyn 1996) than those of typical clouds in the disk region $(T \sim 15 \mathrm{~K})$. The above soft radiation environment can partly account for such relatively high cloud temperatures seen in the Galactic center region. Spaans et al. (1994) pointed out that gas temperature at the $\mathrm{C} / \mathrm{CO}$ transition zone becomes higher when radiation effective temperature is lower, because the $\mathrm{C} / \mathrm{CO}$ transition layers shift toward the surface of the PDR, as mentioned above; according to the model calculation, $T_{\text {gas }} \sim 10 \mathrm{~K}$ for $T_{\text {eff }}=30000 \mathrm{~K}$, but $T_{\text {gas }} \sim 32 \mathrm{~K}$ for $T_{\text {eff }}=6000 \mathrm{~K}$ at the C/CO transition zone with $n=10^{3} \mathrm{~cm}^{-3}$ and $G_{0}=10^{3}$. Towards the edge of a PDR, gas temperature becomes higher, which does not fall below $125 \mathrm{~K}$ even for $T_{\text {eff }}=6000 \mathrm{~K}$; the photoelectric heating rate at $6000 \mathrm{~K}$ is only a factor 3 lower than that at $30000 \mathrm{~K}$, because the photoelectric heating by polycyclic aromatic hydrocarbons is effective in cool PDRs (Spaans et al. 1994).

This effect is supported by observations of a series of CO lines. The CO lines are expected to be bright in PDRs. Furthermore, the model shows that the ratio of $[\mathrm{C} \mathrm{II}] / \mathrm{CO}$ is a good indicator of $T_{\text {eff }}$; for example, the ratio $[\mathrm{C} \mathrm{II}] / \mathrm{CO}\left(J_{\max }\right)=$ 1413,298 , and 6.3 for an effective temperature of $30000 \mathrm{~K}$ $\left(J_{\max }=3\right), 10000 \mathrm{~K}\left(J_{\max }=3\right)$, and $6000 \mathrm{~K}\left(J_{\max }=4\right)$, respectively. This results also showed that the value of the maximum $\left(J_{\max }\right)$ shifted to the upper rotational level in the soft radiation field. In fact, observations showed that the CO lines are significantly brighter in the Galactic center (Fixsen et al. 1999; Bennett et al. 1994); the ratio of $[\mathrm{CII}] / \mathrm{CO}\left(J_{\max }\right)$ is $49.4\left(J_{\max }=4\right)$ for the Galactic center region and $298\left(J_{\max }=3\right)$ for the disk region, which is quite consistent with our results of $T_{\text {eff }}=6600 \mathrm{~K}$ and $8600 \mathrm{~K}$ for the Galactic center and the disk region, respectively. Additionally, $J_{\max }$ move to upper rotational level in the Galactic center.

Hence, the soft radiation field, inferred from our observation, can be one of the sources for the higher temperature of molecular clouds in the Galactic center region. We do not rule out other possibilities such as the dissipation of turbulence (Morris \& Serabyn 1996), to explain the existence of higher temperature molecular clouds.

\subsubsection{Other mechanisms}

We discuss the possibility of contribution of shocks and X-rays to the low [C II]/FIR ratio in the Galactic center region. Since the observed low [C II]/FIR ratio is a large-scale trend, the shocks would have to have a large filling factor to dominate the observations of such a large area of the Galactic center region.

On the other hand, diffuse X-ray emission in the Galactic center region has also been detected (e.g. Koyama et al. 1996), which could affect physical conditions in the Galactic center. X-rays can certainly play a role; X-ray-dominated regions (XDRs) can account for the high temperatures of gas more easily because gas is heated more effectively in XDRs than in PDRs. Rodríguez-Fernández et al. (2004), however, contradicted the possibility that XDRs clouds to explain the warm gas. In the case of XDRs, $\mathrm{C}^{+}$are present throughout clouds, while they are only in the near-edge regions of clouds in PDRs (Meijerink $\&$ Spaans 2005). Therefore, the contribution of XDRs would rather enhance the total [C II] line intensity in the Galactic center region, which obviously contradicts the observational result. In addition, an XDR model predicts that the $[\mathrm{OI}] /[\mathrm{C} \mathrm{II}]$ line ratio for low density $\left(\leq 10^{3} \mathrm{~cm}^{-3}\right)$ XDRs $(\geq 1)$ is higher than that for low density PDRs ( $\geq 0.1$; Meijerink et al. 2007); our results are $0.1-0.6$. Hence, XDRs are not favorable to explain the low $[\mathrm{C}$ II $] /$ FIR ratio.

\subsection{Star formation history in the Galactic center region}

In the previous subsection, we show that soft interstellar radiation fields are responsible for the systematically low $[\mathrm{C}$ II]/FIR ratios in the Galactic center region. Here, we discuss what causes the soft radiation in terms of a star-formation history. We consider two simple star-formation models; one is the model where there was a starburst in the past with no current star-formation activity (model 1), the other is the model where star-formation activities continue up to present (model 2). These models are rather extreme cases, and real cases should be somewhere between the two models.

We assume a power-law initial mass function (IMF):

$\mathrm{d} N=N_{0} m^{-\alpha} \mathrm{d} m$,

where $m$ is the stellar mass, $\mathrm{d} N$ is the number of stars produced during the starburst per unit time in a mass interval $\mathrm{d} m$, and $N_{0}$ is the normalizing constant. For the model 1, a starburst is assumed to have begun $t_{0}$ years ago and to have continued during a period, $t_{\text {burst }}$, at a constant starburst rate. We determine $N_{0}$ from the present bolometric luminosity $L_{\mathrm{bol}}$,

$$
L_{\text {bol }}=\left\{\begin{array}{lr}
\int \ell(m) N_{0} m^{-\alpha} t_{\text {burst }} \mathrm{d} m & \left(\tau(m)>t_{0}\right), \\
\int \ell(m) N_{0} m^{-\alpha}\left(\tau(m)-t_{0}+t_{\text {burst }}\right) \mathrm{d} m & \left(t_{0}>\tau(m)>t_{0}-t_{\text {burst }}\right), \\
0 & \left(t_{0}-t_{\text {burst }}>\tau(m)\right),
\end{array}\right.
$$

where $\tau(m)$ is the lifetime of a star with $m$. We assume that a stellar mass ranges from 0.08 to $30 M_{\odot}, \alpha$ is the Salpeter value, 2.35 , and $\tau(m)=10.02-3.57 \log m+0.9(\log m)^{2}($ Larson 1974). The mass-luminosity relation $\ell(m)$ is adopted from Drilling \& Landolt (1999). $L_{\text {bol }}$ is set to be equal to the observed FIR luminosity, where we neglect the contribution of pre- and post-mainsequence stars. We made calculation for value of $t_{0}$ of $1 \times 10^{6}$, $3 \times 10^{6}, 1 \times 10^{7}, 3 \times 10^{7}, 1 \times 10^{8}, 3 \times 10^{8}$, and $1 \times 10^{9} \mathrm{yr}$; all the stars are still alive in $1 \times 10^{6} \mathrm{yr}$, while most of them die in 
Table 4. Dependence of $T_{\mathrm{c}}$, stellar radiation color temperature on $t_{0}$, the time when a starburst started, derived from the two star formation models.

\begin{tabular}{ccc}
\hline \hline & $t_{0}=1 \times 10^{9} \mathrm{yr}$ & $t_{0}=1 \times 10^{7} \mathrm{yr}$ \\
\hline Model 1 & $T_{\mathrm{c}} \sim 8000 \mathrm{~K}$ & $T_{\mathrm{c}} \sim 26000 \mathrm{~K}$ \\
Model 2 & $T_{\mathrm{c}} \sim 31000 \mathrm{~K}$ & $T_{\mathrm{c}} \sim 32000 \mathrm{~K}$ \\
\hline
\end{tabular}

$1 \times 10^{9} \mathrm{yr}$ with masses higher than $1 M_{\odot}$. We assume that $t_{\text {burst }}$ ranges from $3 \times 10^{5}$ to $1 \times 10^{7}$, depending on $t_{0}$.

To predict the stellar radiation temperature, we calculate the number of H-ionizing photons $\left(N_{\mathrm{UV}} ; E \geq 13.6 \mathrm{eV}\right)$ and C-ionizing photons $\left(N_{\mathrm{CII}} ; 13.6 \geq E \geq 11.3 \mathrm{eV}\right)$ per unit time produced by stars in our model;

$$
\begin{aligned}
N_{\mathrm{UV}}= & \int n_{\mathrm{uv}}(m) N_{0} m^{-\alpha} t_{\text {burst }} \mathrm{d} m \\
& +\int n_{\mathrm{uv}}(m) N_{0} m^{-\alpha}\left(\tau(m)-t_{0}+t_{\text {burst }}\right) \mathrm{d} m \\
N_{\mathrm{CII}}= & \int n_{\mathrm{cii}}(m) N_{0} m^{-\alpha} t_{\text {burst }} \mathrm{d} m \\
& +\int n_{\text {cii }}(m) N_{0} m^{-\alpha}\left(\tau(m)-t_{0}+t_{\text {burst }}\right) \mathrm{d} m .
\end{aligned}
$$

In these equations, $n_{\mathrm{uv}}(m)$ and $n_{\mathrm{cii}}(m)$ are the numbers of $\mathrm{H}-$ and C-ionizing photons emitted by a single star per unit time as a function of a stellar mass $m$, respectively, where stellar emission is assumed to have spectra presented by the stellar atmosphere model by Kurucz (1991).

For the other model (model 2), star-formation activities are assumed to have begun $t_{0}$ years ago and to continue up to present at a constant rate. $L_{\text {bol }}$ in the Eq. (4) is then expressed as

$L_{\mathrm{bol}}= \begin{cases}\int \ell(m) N_{0} m^{-\alpha} t_{0} \mathrm{~d} m & \left(\tau(m)>t_{0}\right), \text { and } \\ \int \ell(m) N_{0} m^{-\alpha} \tau(m) \mathrm{d} m & \left(\tau(m)<t_{0}\right) .\end{cases}$

We also calculate $N_{\mathrm{UV}}$ and $N_{\mathrm{CII}}$ in this model from the following equations.

$$
\begin{aligned}
& N_{\mathrm{UV}}=\int n_{\mathrm{uv}}(m) N_{0} m^{-\alpha} t_{0} \mathrm{~d} m+\int n_{\mathrm{uv}}(m) N_{0} m^{-\alpha} \tau(m) \mathrm{d} m \\
& N_{\mathrm{CII}}=\int n_{\mathrm{cii}}(m) N_{0} m^{-\alpha} t_{0} \mathrm{~d} m+\int n_{\mathrm{cii}}(m) N_{0} m^{-\alpha} \tau(m) \mathrm{d} m .
\end{aligned}
$$

We derived the color temperature of stellar radiation $\left(T_{\mathrm{c}}\right)$ from $N_{\mathrm{UV}} / N_{\mathrm{CII}}$ for each of the above star-formation models. For the model $1, T_{\mathrm{c}}$ depends strongly on $t_{0}$ but less on $t_{\text {burst }}$. Table 4 shows the dependence of $T_{\mathrm{c}}$ on $t_{0}$ for the two star-formation models. By setting the observed $T_{\text {eff }}$ equal to the calculated $T_{\mathrm{c}}$, we have found that the observational result supports the case of the model 1 with $t_{0}=1 \times 10^{9} \mathrm{yr}$, which implies that the starformation activities in the Galactic center region are currently low, although they were intense $\sim 1$ Gyr ago. The exact age depends upon the assumed IMF, but general trends do not change even if we change the IMF.

Mezger et al. (1999) and Philipp et al. (1999) showed an overabundance of evolved stars in the Galactic center region of $650^{\prime \prime} \times 710^{\prime \prime}$ area, which can be explained by a strong increase in star formation rate $10^{7}-10^{8}$ years ago. Near-infrared spectroscopy of asymptotic giant branch and cool supergiant stars in the central $5 \mathrm{pc}$ of the Galaxy support that the Galactic center is currently in a more quiescent state than in the recent past (Gyr to 10 Gyr; Blum et al. 2003). In addition, Odenwald \& Fazio (1984) showed that the average infrared excess of an FIR extended component is 50 in the Galactic center, which implies an even later population of stars in the extended component than in FIR discrete sources that have an average value of 16 . These results indicate the current star-formation activity in the Galactic center region is inactive, and thus consistent with our star-formation history model.

\subsection{Relations with other galaxies}

Malhotta et al. (2001) analyzed 60 normal, star-forming galaxies and reported that one-third of the galaxies in the sample show a smooth decline in $L_{\left[\mathrm{C}_{\text {II }}\right]} / L_{\mathrm{FIR}}$ with increasing $F_{60} / F_{100}$. Malhotta et al. concluded that this is due to increased positive grain charge in the warmer and more active galaxies, which lead to less efficient heating by photo-electrons from dust grains. This conclusion cannot be applied to the Galactic center, since the tendency which Malhotta et al. found for starburst galaxies is different from what we found towards the Galactic center; $L_{[\mathrm{C} \text { II] }} / L_{\mathrm{FIR}}$ is always low regardless of $F_{60} / F_{100}$ or $G_{0}$ (see Fig. 5).

Luhman et al. (1998, 2003) found that ultra-luminous infrared galaxies (ULIRGs) showed order of magnitude deficit (compared to normal and starburst galaxies) in the strength of the [C II] line relative to the far-infrared dust continuum emission. In ULIRGs, however, the low [C II]/FIR ratio is not likely to be attributed to the soft radiation field. As pointed out by Luhman et al. (2003), if the current radiation field were soft, the ULIRGs would have to be more luminous at an earlier age. However, such extremely luminous galaxies have not been found in the local universe. Hence, the [C II] line deficit observed in ULIRGs is mostly attributed to a very strong radiation field irradiating a PDR cloud rather than the soft radiation field. In the Galactic center of our own, the [C II]/FIR ratio decreases not only in high$G_{0}$ region but also in low- $G_{0}$ regions. Thus, the cause of the low $[\mathrm{C}$ II]/FIR ratio is different between the Galactic center of our own and ULIRGs.

\subsection{HII regions in the Galactic center region}

Judging from the above consistency of our observational results with the BICE data, most of the large-scale FIR emission in the Galactic center region is likely to originate from PDRs. Nevertheless, we also detect [O III] $52 \mu \mathrm{m}$, [O III] $88 \mu \mathrm{m}$, and [N II] $122 \mu \mathrm{m}$ lines suggesting the presence of weak H II regions along the lines of sight. By using these emission lines, we discuss the nature of the $\mathrm{H}$ II regions in the Galactic center region.

We estimate electron densities in the HII regions by using the ratio of the two [O III] emission lines at 52 and $88 \mu \mathrm{m}$ (Emery \& Kessler 1984). Under the same assumption as adopted in the case of the [OI] lines except for the gas temperature of $10000 \mathrm{~K}$, the line optical depths for both lines are as small as $\tau_{52} \sim 0.07$ and $\tau_{88} \sim 0.03$ in the Galactic center region, thus justifying the optically thin assumption in calculating electron densities. Figure 6 shows the electron densities obtained from the [O III] $52 \mu \mathrm{m} / 88 \mu \mathrm{m}$ ratios, most of which are of the order of $10^{2} \mathrm{~cm}^{-3}$. The averaged electron density in the Galactic center region is $\sim 480 \pm 300 \mathrm{~cm}^{-3}$, while that in the Galactic disk is $100-400 \mathrm{~cm}^{-3}$. Hence, there is no significant difference in the electron density between the Galactic center and disk regions. Our result is also consistent with those of Rodríguez-Fernández et al. (2001) and Goicoechea et al. (2004), which showed the electron densities near the Pistol and the Sgr B2 regions are $10^{1.8-2.6}$ and $10^{1-3} \mathrm{~cm}^{-3}$, respectively. 


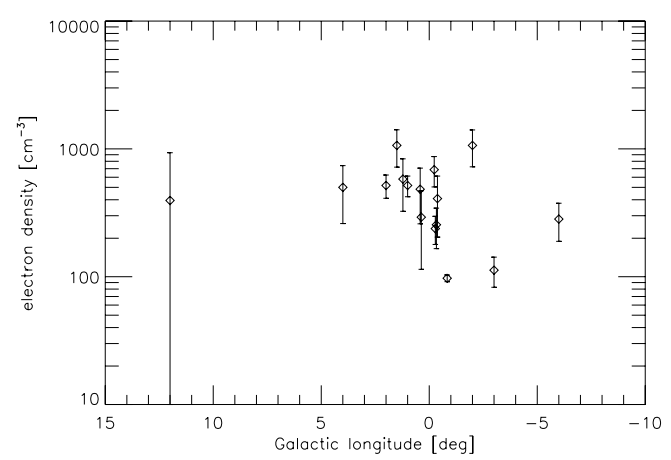

Fig. 6. Distribution of electron densities derived from [O III] 52 and $88 \mu \mathrm{m}$ emission lines along the Galactic plane.

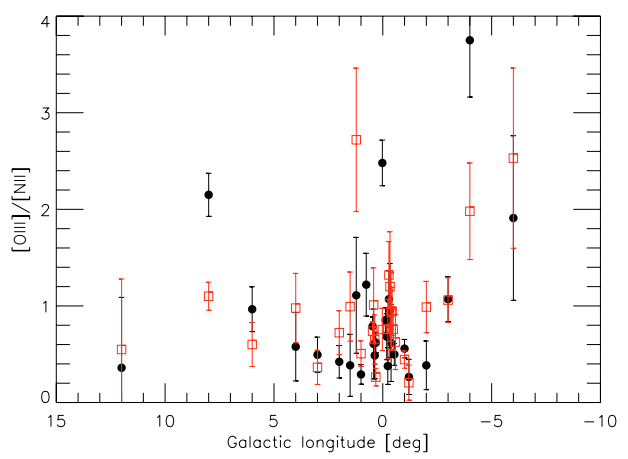

Fig. 7. $[\mathrm{O} \mathrm{III}] /[\mathrm{N}$ II] ratios observed by the ISO/LWS. The open squares indicate $[\mathrm{O}$ III $] 52 \mu \mathrm{m} /[\mathrm{N}$ II $]$ ratios, while the filled circles indicate [O III] $88 \mu \mathrm{m} /[\mathrm{N}$ II $]$.

In Fig. 7, we show the observed ratios of [O III] $52 \mu \mathrm{m} /$ [N II] $121 \mu \mathrm{m}$ and [O III] $88 \mu \mathrm{m} /[\mathrm{N}$ II $] 121 \mu \mathrm{m}$; they do not show a clear difference between the Galactic center and the disk regions. We evaluate the color temperature of the radiation field from the $[\mathrm{O} \mathrm{III}] /[\mathrm{N} \mathrm{II}]$ ratios. In the same manner as performed above, the [N II] line is estimated to have the optical depth of $\tau_{122} \sim 0.01$ with relative abundance of $\mathrm{N}, \delta_{\mathrm{N}}=$ $1 \times 10^{-4}$ (Sternberg \& Dalgarno 1995) and thus is optically thin. We calculate the $[\mathrm{O}$ III $] /[\mathrm{N}$ II] ratios adopting the electron densities obtained above under the assumption that these lines are emitted from the same region. The ratios of the $\mathrm{O}^{++}$to the $\mathrm{N}^{+}$densities are derived from the numbers of the photons ionizing corresponding species; photons are produced by stars in the above model where $T_{\mathrm{c}}$ is a free parameter. Figure 8 shows the $[\mathrm{O} \mathrm{III}] /[\mathrm{N} \mathrm{II}]$ ratios calculated with $T_{\mathrm{c}}=40000 \mathrm{~K}$, which are in fair agreement with the overall observational results. Hence, the $\mathrm{H}$ II regions in the Galactic center region appear to have no particular differences from other H II regions in disk regions.

\section{Summary and conclusions}

We have analyzed dust continuum and fine-structure line emission data in the Galactic center region obtained by ISO. Our results show that the ratio of the [C II] to the FIR continuum emission is systematically low whereas that of the [O I] to the FIR is almost constant towards the Galactic center; the former result is consistent with that of the previous BICE [C II]-line mapping (Nakagawa et al. 1995, 1998). We have succeeded in unambiguously identifying the cause of the low [C II]/FIR ratio by multiple-line spectroscopy. We have derived physical parameters of the ISM in the Galactic center region by using the PDR model (Spaans et al. 1994), which takes account of an effective

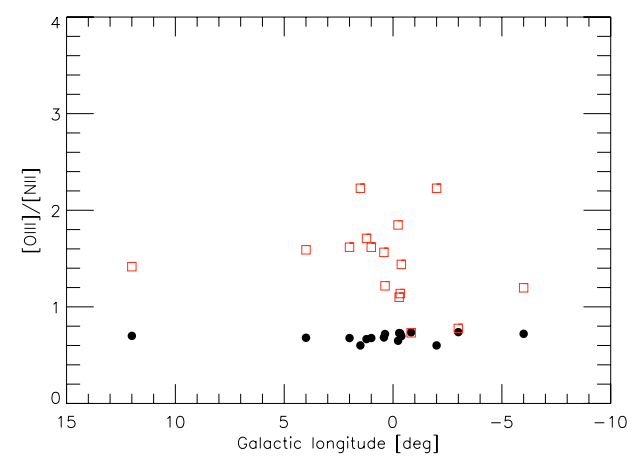

Fig. 8. $[\mathrm{O} \mathrm{III}] /[\mathrm{N}$ II $]$ ratios calculated with $T_{\text {eff }}=40000 \mathrm{~K}$. The open squares indicate [O III] $52 \mu \mathrm{m} /[\mathrm{N}$ II] ratios, while the filled circles indicate $[\mathrm{O}$ III $] 88 \mu \mathrm{m} /[\mathrm{N}$ II $]$ ratios.

temperature of interstellar radiation field. The results show that the effective temperatures are significantly low in the Galactic center region. We conclude that the suppression of the [C II]/FIR ratio toward the Galactic center is not predominantly due to either strong radiation field or high-density PDRs, but most probably due to a soft radiation field in the Galactic center region. The soft radiation field may even solve the problem of the systematic difference in temperature of clouds between the Galactic center and disk regions.

To discuss physical implications of the soft radiation field in the Galactic center region, we have introduced the two starformation models, where we calculate the numbers of $\mathrm{H}$ - and $\mathrm{C}$-ionizing photons produced by stars and derived color temperatures from ratios of these photons. As a result, the observed effective temperature supports the model where star-formation activities in the Galactic center region started a long time ago $(1 \times$ $10^{9} \mathrm{yr}$ ) and turned off in the past. Although a dominant fraction of the observed FIR emission is likely to originate from PDRs, we also detect [O III] and [N II] lines, suggestive of the presence of $\mathrm{H}$ II regions, which are found to have electron densities and effective temperatures similar to those in other $\mathrm{H}$ II regions in ordinary disk regions. We conclude that dominant sources of the FIR luminosity are not likely to be young OB stars but rather cool stars, $\mathrm{K}$ and $\mathrm{M}$ giants, which implies that current starformation activity is rather low in the Galactic center region.

Acknowledgements. The ISO Spectral Analysis Package (ISAP) is a joint development by the LWS and SWS Instrument teams and the ISO data center. Contributing institutes are CESR, IAS, IPAC, MPE, RAL and SRON.

\section{References}

Bennett, C. L., Fixsen, D. J., Hinshaw, G., et al. 1994, ApJ, 434, 587 Blum, R. D., Ramírez, S. V., Sellgren, K., \& Olsen, K. 2003, ApJ, 597, 323 Cotera, A. S., Erickson, E. F., Colgan, S. W. J., et al. 1996, ApJ, 461, 750 Cotera, A. S., Colgan, S. W. J., Simpson, J. P., \& Rubin, R. H. 2005, ApJ, 622, 333

Cox, P., \& Laureijs, R. 1989, in The Center of the Galaxy, ed. M. Morris (Kluwer: Dordrecht), IAU Symp., 136, 121

Crawford, M. K., Genzel, R., Townes, C. H., \& Watson, D. M. 1985, ApJ, 291, 755

Draine, B. T. 1978, ApJS, 36, 595

Drilling, J. S., \& Landolt, A. U. 1999, in Allen's Astrophysical Quantities 4th edn., ed A. Cox (New York: Springer), 381

Emery, R. J., \& Kessler, M. F. 1984, in Galactic and extragalactic infrared spectroscopy, ed. M. F. Kessler, \& J. P. Phillips, 289 
Figer, D. F., Kim, S. S., Morris, M., et al. 1999, ApJ, 525, 750

Fixsen, D. J., Bennett, C. L., \& Mather, J. C. 1999, ApJ, 526, 207

Genzel, R. 1991, in The Galactic Interstellar Medium, ed. W. B. Burton, B. G. Elmegreen, \& R. Genzel (Berlin: Springer-Verlag), 275

Genzel, R., Crawford, M. K., Townes, C. H., \& Watson, D. M. 1985, ApJ, 297, 766

Goicoechea, J. R., Rodríguez-Fernández, N. J., \& Cernicharo, J. 2004, ApJ, 600, 214

Gry, C., Swinyard, B., Harwood, A., et al. 2003, in The ISO HANDBOOK, volume III, ver. 2.1 (ESA: Noordwijk)

Habing, H. J. 1968, Bull. Astr. Inst. Netherlands, 19, 421

Handa, T., Sofue, Y., Nakai, N., et al. 1987, PASJ, 39, 709

Helou, G., Khan, I. R., Malek, L., \& Boehmer, L. 1988, ApJS, 68, 151

Hollenbach, D. J., Takahashi, T., \& Tielens, A. G. G. M. 1991, ApJ, 377, 192

Hollenbach, D. J., \& Tielens, A. G. G. M. 1997, ARA\&A, 35, 179

Koyama, K., Maeda, Y., Sonobe, T., et al. 1996, PASJ, 48, 249

Kurucz, R. L. 1991, in Proceedings of the Workshop on Precision Photometry: Astrophysics of the Galaxy, ed. A. C. Davis Philip, A. R. Upgren, \& K. A. James (Schenectady: Davis press), 27

Larson, R. B. 1974, MNRAS, 166, 585

Lis, D. C., Serabyn, E., Zylka, R., \& Li, Y. 2001, ApJ, 550, 761

Luhman, M. L., Satyapal, S., Fischer, J., et al. 1998, ApJ, 504, L11

Luhman, M. L., Satyapal, S., Fischer, J., et al. 2003, ApJ, 594, 758

Malhotra, S., Kaufman, M. J., Hollenbach, D., et al. 2001, ApJ, 561, 766

Mathis, J. S. 1990, ARA\&A, 28, 37
Meijerink, R., \& Spaans, M. 2005, A\&A, 436, 397

Meijerink, R., Spaans, M., \& Israel, F. P. 2007, A\&A, 461, 793

Mezger, P. G., Zylka, R., Salter, C. J., et al. 1989, A\&A, 209, 337

Mezger, P. G., Zylka, R., Philipp, S., \& Launhardt, R. 1999, A\&A, 348, 457

Morris, M., \& Serabyn, E. 1996, ARA\&A, 34, 645

Nakagawa, T., Doi, Y., Yui, Y. Y., et al. 1995, ApJ, 455, L35

Nakagawa, T., Yui, Y. Y., Doi, T., et al. 1998, ApJS, 115, 259

Odenwald, S. F., \& Fazio, G. G. 1984, ApJ, 283, 601

Oort, J. H. 1977, ARA\&A, 15, 295

Philipp, S., Zylka, R., Mezger, P. G., et al. 1999, A\&A, 348, 768

Pierce-Price, D., Richer, J. S., Greaves, J. S., et al. 2000, ApJ, 545, L121

Rodríguez-Fernández, N. J., Martín-Pintado, J., \& de Vicente, P. 2001, A\&A, 377,631

Rodríguez-Fernández, N. J., Martín-Pintado, J., Fuente, A., \& Wilson, T. L. 2004, A\&A, 427, 217

Rodríguez-Fernández, N. J., \& Martín-Pintado, J. 2005, A\&A, 429, 923

Spaans, M., Tielens, A. G. G. M., van Dishoeck, E. F., \& Bakes, E. L. O. 1994, ApJ, 437, 270

Sternberg, A., \& Dalgarno, A. 1995, ApJS, 99, 565

Swinyard, B. M., Clegg, P. E., Ade, P. A. R., et al. 1996, A\&A, 315L, 43

Tielens, A. G. G. M. 2005, in The physical and chemistry of the Interstellar Medium (Cambridge: Cambridge university press), 317

Tielens, A. G. G. M., \& Hollenbach, D. J. 1985, ApJ, 291, 722

van Loon, J. Th., Gilmore, G. F., Omont, A., et al. 2003, MNRAS, 338, 857

Wolfire, M. G., Tielens, A. G. G. M., \& Hollenbach, D. J. 1990, ApJ, 358, 116 